\title{
COMMUNICABLE DISEASES, NSW: DECEMBER 2001
}

\section{TRENDS}

Notifications of communicable disease received through to October 2001 are summarised in Table 3 and Figure 1. Of note are the continuing epidemic of pertussis occurring throughout the State, and a mild resurgence of hepatitis A (12 cases were reported in South Eastern Sydney in October, but no links were identified among them).

\section{AUSTRALIAN CHILDHOOD IMMUNISATION REGISTER-QUARTERLY REPORT}

Table 1 reports immunisation coverage by area health service for children aged 12 months to less than 15 months. These data refer to three different cohorts of children whose age has been calculated 90 days before data extraction. The information contained in each of the reports has been extracted from the Australian Childhood Immunisation Register (ACIR) and may not reflect actual coverage due to under-reporting.

\section{TABLE 1}

IMMUNISATION COVERAGE BY AREA HEALTH SERVICE FOR CHILDREN AGED 12 MONTHS TO LESS THAN 15 MONTHS

\begin{tabular}{|lccc|}
\hline $\begin{array}{l}\text { Area Health } \\
\text { Service }\end{array}$ & $\begin{array}{c}\text { 30 June } \\
\text { 2001 }\end{array}$ & $\begin{array}{c}\text { 30 Sept } \\
\mathbf{2 0 0 1}\end{array}$ & $\begin{array}{c}\text { 31 Dec } \\
\mathbf{2 0 0 1}\end{array}$ \\
\hline Central Coast & 94 & 93 & 94 \\
Central Sydney & 91 & 89 & 87 \\
Hunter & 94 & 96 & 93 \\
Illawarra & 92 & 93 & 91 \\
Northern Sydney & 90 & 89 & 89 \\
South Eastern Sydney & 89 & 89 & 89 \\
South Western Sydney & 92 & 90 & 89 \\
Wentworth & 92 & 92 & 91 \\
Western Sydney & 89 & 90 & 89 \\
Far West & 87 & 92 & 94 \\
Greater Murray & 93 & 93 & 93 \\
Macquarie & 93 & 92 & 95 \\
Mid North Coast & 91 & 91 & 88 \\
Mid Western & 90 & 92 & 92 \\
New England & 92 & 92 & 94 \\
Northern Rivers & 86 & 86 & 84 \\
Southern & 91 & 91 & 89 \\
NSW & 91 & 91 & 91 \\
\hline
\end{tabular}

\section{ASSUMING THE WORST: RESPONDING TO EPISODES OF SUSPECTED BIOLOGICAL TERRORISM}

\section{A. Leask, V. Delpech, M. Boomer, E. Mitchell, B. O'Sullivan}

In mid-October 2001, the NSW Department of Health received the first reports of what emerged as an epidemic of exposures to powders mistakenly thought to contain anthrax spores. These incidents involved individuals who received packages that contained powder or had a suspicious address, or individuals who encountered suspicious powders in unexpected situations. Some incidents apparently had malicious intent while others clearly had an innocuous cause. These events began soon after reports from the United States of individuals who had developed anthrax infections following the receipt of mail that contained powdered anthrax spores.

To coordinate the responses to these threats, the NSW Government re-opened the Police Operations Centre, a communications facility that was last used during the Sydney 2000 Olympic and Paralympic Games. The NSW Department of Health placed a liaison officer in the Police Operations Centre. Several public health officers from the NSW Public Health Officer Training Program, and other staff from the NSW Department of Health, were also seconded to assist. Contact details of people involved in suspicious powder incidents were matched to the results of analyses of the specimens taken from these incidents. The results were distributed to public health units who contacted the victims to inform them of the results.

The responsibility for the management of such incidents is detailed in the NSW Department of Health's HAZMAT Plan, a subsection of the NSW State Disaster Plan (Displan). The role of the area health services included the provision of medical, mental health, and public health services. The police, ambulance and fire brigade also had essential roles in responding to these events.

Public health units were also asked to place laboratories and emergency departments on alert for patients presenting with suspicious illnesses. Chief Health Officers around Australia alerted all divisions of general practice to be vigilant for suspicious illnesses in their patients. Australia was not considered to be at serious risk of terrorist attack; therefore the use of vaccines and prophylactic antibiotics, and the diagnostic testing of victims, was strongly discouraged.

Fact sheets on anthrax and material addressing these incidents were prepared and distributed to public health units. These were developed to help inform and reassure the general public, especially those individuals who had been involved in an incident. A recorded telephone message service on (02) 94245908 was also established to provide information.

Between 16 October and 12 November, 463 samples were submitted to the police forensics laboratory for analysis. These samples were collected from incidents that involved approximately 1500 people. To date no sample has tested positive for anthrax spores.

In the absence of a positive laboratory test for anthrax spores, or a case of anthrax, the main role for NSW Health has been to mitigate the fear associated with these events. Efforts have focused on returning the results of laboratory investigations to the individuals exposed as swiftly as possible. 


\section{TABLE 2}

CHARACTERISTICS OF PEOPLE DIAGNOSED WITH HIV INFECTION AND AIDS, OR WHO HAVE DIED FROM AIDS, NSW, 1981 TO SEPTEMBER 2001

\begin{tabular}{|c|c|c|c|c|c|c|c|c|c|c|c|c|c|c|c|c|c|c|c|c|c|c|}
\hline \multirow{3}{*}{ Characteristic } & \multicolumn{6}{|c|}{ All cases (1981-2000) } & \multicolumn{6}{|c|}{$1991-2000$} & \multicolumn{6}{|c|}{ Jan-Sep 2001} & \multirow{3}{*}{$\begin{array}{l}\text { by } \\
\text { Year }\end{array}$} & \multirow[b]{3}{*}{ HIV } & \multirow{3}{*}{ AIDS } & \multirow{3}{*}{$\begin{array}{c}\text { AIDS } \\
\text { Deaths }\end{array}$} \\
\hline & \multicolumn{2}{|c|}{ HIV } & \multicolumn{2}{|c|}{ AIDS } & \multirow{2}{*}{$\begin{array}{c}\text { AIDS } \\
N\end{array}$} & \multirow{2}{*}{$\begin{array}{c}\text { Deaths } \\
\%\end{array}$} & \multicolumn{2}{|c|}{ HIV } & \multicolumn{2}{|c|}{ AIDS } & \multirow{2}{*}{$\begin{array}{c}\text { AIDS D } \\
\quad N\end{array}$} & \multirow{2}{*}{$\begin{array}{c}\text { Deaths } \\
\quad \%\end{array}$} & \multicolumn{2}{|c|}{ HIV } & \multicolumn{2}{|c|}{ AIDS } & \multirow{2}{*}{$\begin{array}{c}\text { AIDS } \\
N\end{array}$} & \multirow{2}{*}{$\begin{array}{c}\text { Deaths } \\
\%\end{array}$} & & & & \\
\hline & $N$ & $\%$ & $N$ & $\%$ & & & $N$ & $\%$ & $N$ & $\%$ & & & $N$ & $\%$ & $N$ & $\%$ & & & & & & \\
\hline Sex & & & & & & & & & & & & & & & & & & & & & & \\
\hline Male & 11226 & 92.5 & 4662 & 95.6 & 3195 & 96.3 & 4823 & 92.0 & 3095 & 94.9 & 2237 & 96.2 & 248 & 86.4 & 15 & 93.8 & 8 & 85.7 & 1981 & 1 & 1 & 1 \\
\hline Female & 627 & 5.2 & 203 & 4.2 & 115 & 3.5 & 341 & 6.5 & 157 & 4.8 & 83 & 3.6 & 30 & 10.5 & 1 & 6.3 & 1 & 14.3 & 1982 & 0 & 1 & 0 \\
\hline Other & 278 & 2.3 & 11 & 0.2 & 7 & 0.2 & 78 & 1.5 & 9 & 0.3 & 6 & 0.3 & 9 & 3.1 & 0 & 0.0 & 0 & 0.0 & 1983 & 1 & 3 & 1 \\
\hline Age & & & & & & & & & & & & & & & & & & & 1984 & 205 & 30 & 6 \\
\hline $0-2$ & 38 & 0.3 & 7 & 0.1 & 3 & 0.1 & 22 & 0.4 & 7 & 0.2 & 3 & 0.1 & 0 & 0.0 & 0 & 0.0 & 1 & 0.0 & 1985 & 990 & 91 & 46 \\
\hline $3-12$ & 42 & 0.4 & 12 & 0.3 & 9 & 0.3 & 9 & 0.2 & 6 & 0.2 & 5 & 0.2 & 0 & 0.0 & 0 & 0.0 & 0 & 0.0 & 1986 & 1112 & 160 & 108 \\
\hline $13-19$ & 252 & 2.1 & 15 & 0.3 & 11 & 0.3 & 76 & 1.5 & 5 & 0.2 & 6 & 0.3 & 3 & 1.1 & 0 & 0.0 & 0 & 0.0 & 1987 & 1637 & 248 & 142 \\
\hline $20-29$ & 4095 & 33.8 & 821 & 16.8 & 575 & 17.3 & 1612 & 30.8 & 490 & 15.0 & 392 & 16.9 & 68 & 23.7 & 2 & 12.5 & 0 & 0.0 & 1988 & 1144 & 313 & 137 \\
\hline $30-39$ & 4501 & 37.1 & 2034 & 41.7 & 1353 & 40.8 & 1984 & 37.9 & 1380 & 42.3 & 953 & 41.0 & 108 & 37.6 & 7 & 43.8 & 5 & 71.4 & 1989 & 990 & 347 & 235 \\
\hline $40-49$ & 2170 & 17.9 & 1368 & 28.1 & 948 & 28.6 & 992 & 18.9 & 938 & 28.8 & 677 & 29.1 & 62 & 21.6 & 1 & 6.3 & 2 & 28.6 & 1990 & 809 & 421 & 315 \\
\hline $50-59$ & 683 & 5.6 & 468 & 9.6 & 305 & 9.2 & 353 & 6.7 & 337 & 10.3 & 215 & 9.2 & 18 & 6.3 & 4 & 25.0 & 0 & 0.0 & & & & \\
\hline $60+$ & 245 & 2.0 & 151 & 3.1 & 113 & 3.4 & 120 & 2.3 & 98 & 3.0 & 75 & 3.2 & 10 & 3.5 & 2 & 12.5 & 1 & 0.0 & 1991 & 813 & 438 & 335 \\
\hline Not reported & 105 & 0.9 & 0 & 0.0 & 0 & 0.0 & 74 & 1.4 & 0 & 0.0 & 0 & 0.0 & 18 & 6.3 & 0 & 0.0 & 0 & 0.0 & 1992 & 712 & 427 & 304 \\
\hline Exposure & & & & & & & & & & & & & & & & & & & 1993 & 599 & 468 & 369 \\
\hline Male homo-bisexual & 7040 & 58.0 & 3952 & 81.1 & 2757 & 83.1 & 3345 & 63.8 & 2561 & 78.5 & 1898 & 81.6 & 143 & 49.8 & 13 & 81.3 & 5 & 71.4 & 1994 & 512 & 533 & 410 \\
\hline Male homo-bisexual-IDU & U 267 & 2.2 & 183 & 3.8 & 126 & 3.8 & 166 & 3.2 & 130 & 4.0 & 98 & 4.2 & 10 & 3.5 & 0 & 0.0 & 1 & 14.3 & 1995 & 540 & 463 & 343 \\
\hline Injecting drug use & 357 & 2.9 & 47 & 1.0 & 20 & 0.6 & 160 & 3.1 & 42 & 1.3 & 19 & 0.8 & 10 & 3.5 & 0 & 0.0 & 0 & 0.0 & 1996 & 463 & 356 & 254 \\
\hline Heterosexual & 902 & 7.4 & 376 & 7.7 & 187 & 5.6 & 705 & 13.5 & 325 & 10.0 & 164 & 7.1 & 51 & 17.8 & 2 & 12.5 & 1 & 14.3 & 1997 & 437 & 200 & 110 \\
\hline Haemophila* & 115 & 1.0 & 51 & 1.1 & 45 & 1.4 & 7 & 0.1 & 24 & 0.7 & 28 & 1.2 & 0 & 0.0 & 0 & 0.0 & 0 & 0.0 & 1998 & 415 & 166 & 69 \\
\hline Blood-tissue recipient & 117 & 1.0 & 106 & 2.2 & 90 & 2.7 & 28 & 0.5 & 44 & 1.4 & 43 & 1.9 & 0 & 0.0 & 0 & 0.0 & 0 & 0.0 & 1999 & 388 & 106 & 63 \\
\hline Needle-stick injury & 4 & 0.0 & 0 & 0.0 & 0 & 0.0 & 4 & 0.1 & 0 & 0.0 & 0 & 0.0 & 0 & 0.0 & 0 & 0.0 & 0 & 0.0 & 2000 & 363 & 104 & 69 \\
\hline Vertical & 33 & 0.3 & 14 & 0.3 & 7 & 0.2 & 27 & 0.5 & 12 & 0.4 & 6 & 0.3 & 0 & 0.0 & 0 & 0.0 & 1 & 0.0 & & & & \\
\hline Not stated & 3296 & 27.2 & 147 & 3.0 & 85 & 2.6 & 800 & 15.3 & 123 & 3.8 & 70 & 3.0 & 73 & 25.4 & 1 & 6.3 & 1 & 0.0 & & & & \\
\hline Residence & & & & & & & & & & & & & & & & & & & Jan-Mar 2001 & 92 & 7 & 4 \\
\hline Sydney & 6701 & 55.2 & 4040 & 82.9 & 2767 & 83.4 & 3991 & 76.1 & 2707 & 83.0 & 1951 & 83.9 & 237 & 82.6 & 15 & 93.8 & 9 & 100.0 & April-Jun 2001 & 89 & 9 & 5 \\
\hline Rural & 779 & 6.4 & 678 & 13.9 & 420 & 12.7 & 534 & 10.2 & 522 & 16.0 & 347 & 14.9 & 35 & 12.2 & 1 & 6.3 & 0 & 0.0 & July-Sept 2001 & 106 & - & - \\
\hline Unknown & 4651 & 38.3 & 158 & 3.2 & 130 & 3.9 & 717 & 13.7 & 32 & 1.0 & 28 & 1.2 & 15 & 5.2 & 0 & 0.0 & 0 & 0.0 & Total 2001 & 287 & 16 & 9 \\
\hline Total & 12131 & 100.0 & 4876 & 100.0 & 3317 & 100.0 & 5242 & 100.0 & 3261 & 100.0 & 2326 & 100.0 & 287 & 100.0 & 16 & 100.0 & 9 & 100.0 & Total 1 & 12418 & 4892 & 3326 \\
\hline
\end{tabular}

* Includes people with coagulation disorders 


\section{SURVEILLANCE FOR HUMAN \\ IMMUNODEFICIENCY VIRUS INFECTION, NSW, TO SEPTEMBER 2001}

To the end of September 2001, 287 new diagnoses of HIV infection, 16 cases of AIDS and nine deaths from AIDS were reported to the NSW Department of Health (Table 2). In July 2001, the system for surveillance for HIV infection was revised to enable better tracking of cases. The new system involves initial reporting by the laboratory of positive HIV tests (by de-identified name code) to the Communicable Diseases Surveillance and Control Unit (CDSCU) of the NSW Department of Health. CDSDU staff then collect information about risk factors associated with the case and a history of any previous positive tests. Table 2 includes preliminary data provided by the laboratories. These data will be updated as new information about the cases is received from the treating doctors. The number of people living with - and who have died from-AIDS is likely to be under-reported. Public health units are currently working with clinicians and laboratory staff in each area health service in an annual active surveillance effort to maximise the reporting of AIDS cases. This active surveillance is necessary to ensure accurate management of the epidemic. 


\section{FIGURE 1}

\section{REPORTS OF SELECTED COMMUNICABLE DISEASES, NSW, JANUARY 1996 TO OCTOBER 2001,}

BY MONTH OF ONSET

These are preliminary data: case counts for recent months may increase because of reporting delays. Laboratory-confirmed cases, except for measles, meningococcal disease and pertussis.

\begin{tabular}{|rc|}
\hline \multicolumn{2}{|c|}{ NSW population } \\
Male & $50 \%$ \\
$<5$ & $7 \%$ \\
$5-24$ & $28 \%$ \\
$25-64$ & $52 \%$ \\
$65+$ & $13 \%$ \\
Rural $^{*}$ & $42 \%$ \\
\hline
\end{tabular}
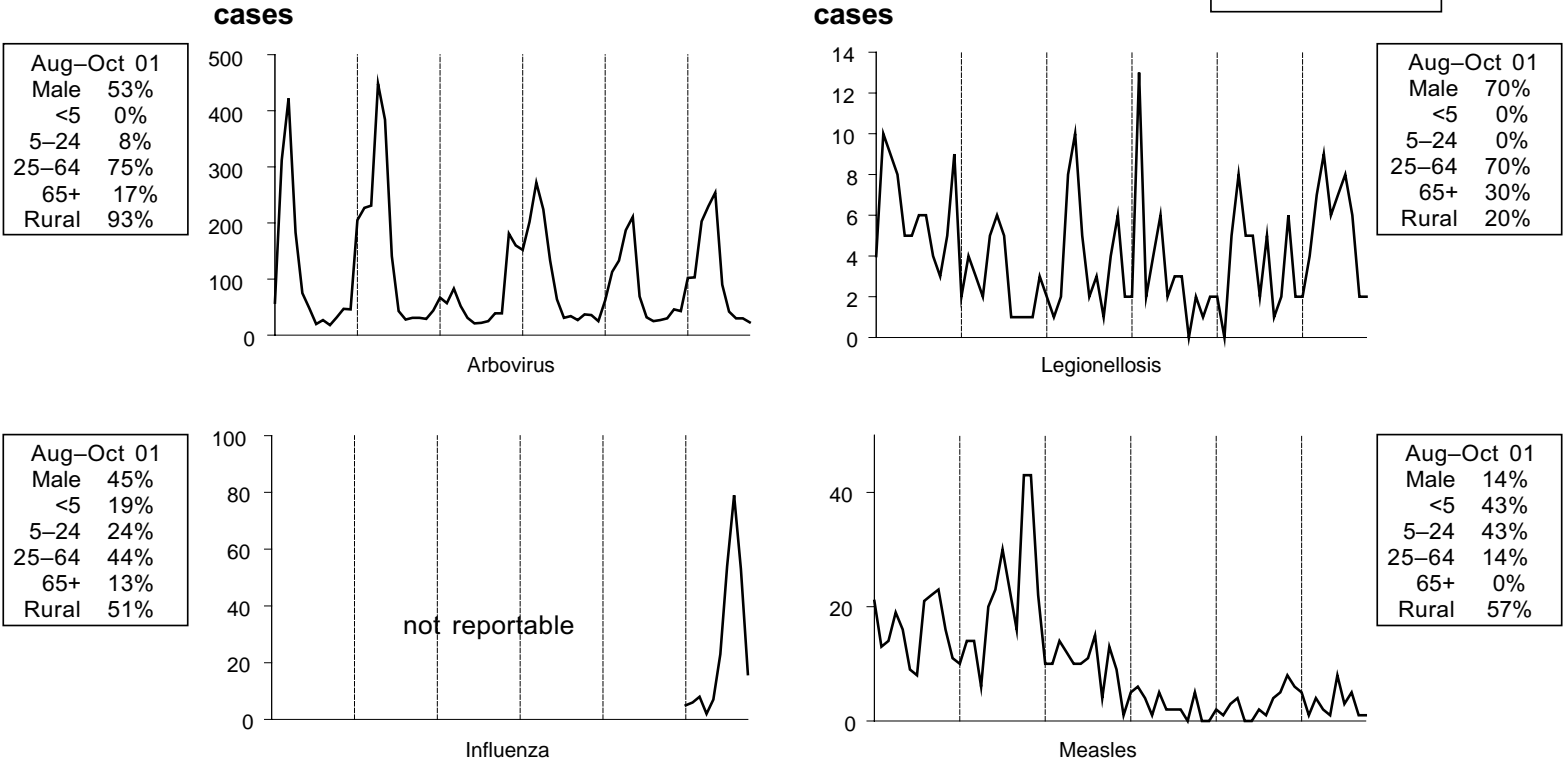

\begin{tabular}{|rc|}
\hline \multicolumn{2}{|c|}{ Aug-Oct 01} \\
Male & $87 \%$ \\
$<5$ & $<1 \%$ \\
$5-24$ & $22 \%$ \\
$25-64$ & $78 \%$ \\
$65+$ & $0 \%$ \\
Rural & $15 \%$ \\
\hline
\end{tabular}
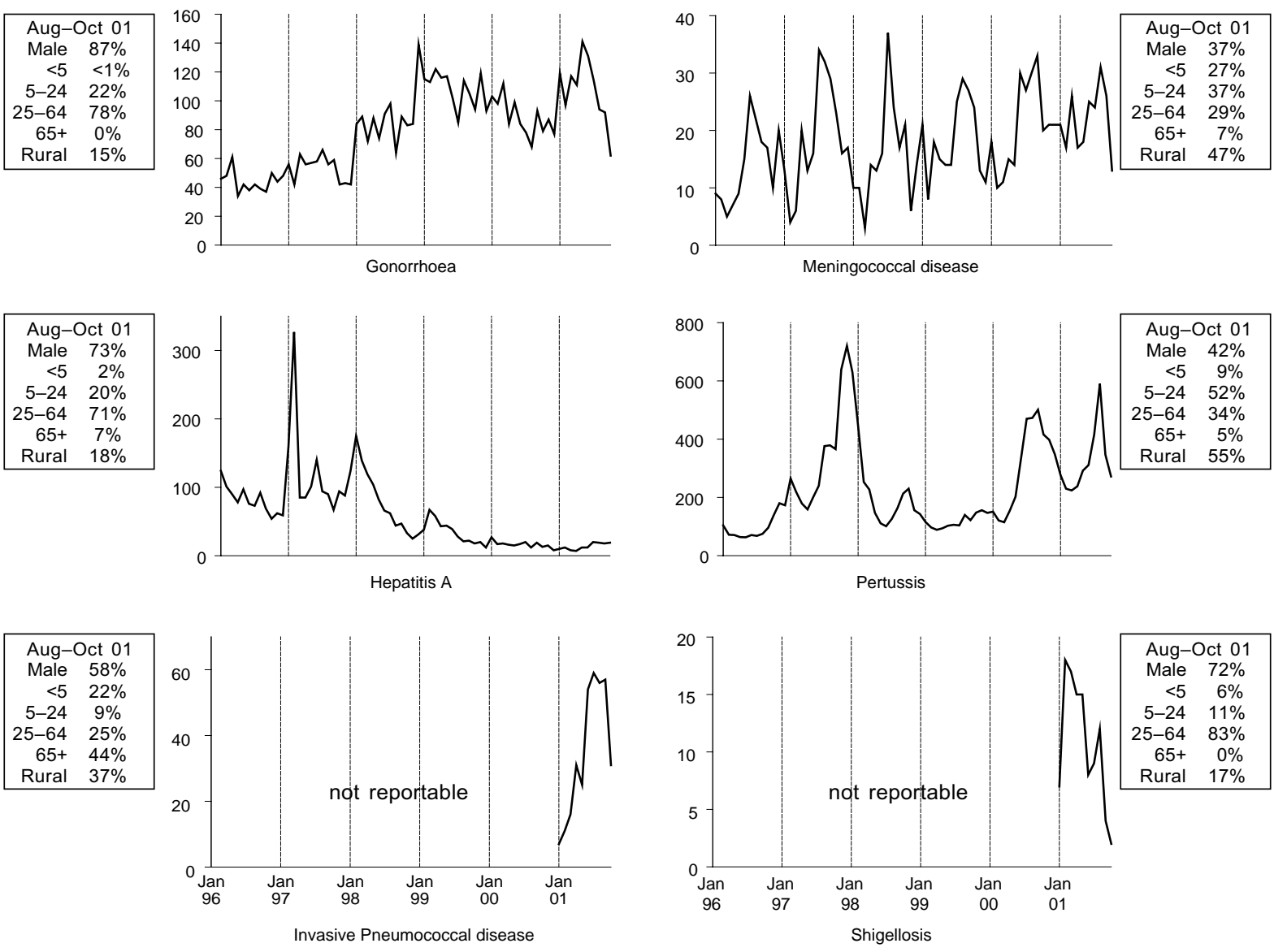

* For definition, see NSW Public Health Bulletin, April 2000 
Hepatitis B - acute viral

Hepatitis B - other

Hepatitis C - acute vira
Hepatitis C - other

Hepatitis D - unspecified

Syphilis

Vector-borne

Arboviral infection $(\mathrm{BFV})^{*}$

Arboviral infection (Other)

Arboviral infection (RRV)*

Malaria*

Zoonoses

Anthrax

Brucellosis*
Leptospirosis*

Lyssavirus*

Psittacosi

\section{Respiratory and other}

Blood lead leve

Invasive pneumococcal infection

Legionella longbeachae infection*

Legionella pneumophila infection

Legionnaires' disease (other)

Leprosy

Meningococcal infection (invasive)

Tuberculosis

\begin{tabular}{|c|c|c|c|c|}
\hline 26 & 19 & 31 & 17 & \\
\hline 22 & 2 & 6 & & \\
\hline 1 & & - & & \\
\hline 61 & 22 & 72 & $\varepsilon$ & \\
\hline
\end{tabular}

Adverse event after immunisation

$H$.influenzae $b$ infection (invasive)

Measles

Mumps*
Pertussi

Pertussis
Rubella*

Tetanus

Faecal-oral

Botulism

Cholera $^{*}$

Cryptosporidiosis ${ }^{\star}$

Gastroenteritis (in an institution)

Gastroenter

Haemolytic uraemic syndrome

Hepatitis A

Hepatitis $E^{*}$
Listeriosis* $^{*}$

Salmonellosis (not otherwise specified)

Shigellosis

Typhoid and paratyphoid*

Verotoxin producing E. col

* lab-confirmed cases only

$\begin{array}{rrrr}1 & - & - & \\ 91 & 27 & 75 & 41\end{array}$

CSA = Cental Sydney Area

$\dagger$ includes cases with unknown postcode 